\title{
Molecular surveillance of healthcare-associated Candida infections in a rehabilitation centre for patients with severe acquired brain injuries
}

Scordino, F1., Galeano, G²., Orlando, M.G²., Barberi, G¹., Giosa, D²., Giuffrè, L²., Marino Merlo, F¹., Criseo, G²., Romeo, O1,2.

${ }^{1}$ IRCCS Centro Neurolesi "Bonino-Pulejo", Messina, Italy

${ }^{2}$ Department of Chemical, Biological, Pharmaceutical and Environmental Sciences, Messina, Italy

Today, infections caused by fungi represent a very important and neglected public health problem as evidenced by over 1.5 million of people supposed die annually worldwide [1]. This mortality rate currently exceeds that of other serious infectious diseases, such as malaria (445.000 deaths in 2016) or HIV/AIDS (1 million deaths in 2016) even if the extent of the global burden of fungal infections appears to be considerably much wider (www.who.int; www.gaffi.org). The impact of these infections on the economy of the countries and their health systems is also enormous, with billions of euros/dollars spent each year because of the burden of healthcare-associated infections (HAls). In Italy, Candida species account for almost $10 \%$ of all ICU-acquired bloodstream infections (BSI) with C. albicans being the most frequently encountered species followed by C. parapsilosis and C. glabrata, C. krusei and C. tropicalis [2]. However, several Italian studies have shown significant regional differences in the prevalence of pathogenic Candida spp., and often non-albicans species (NAC) cause 50\% of the Candida BSI or even outrank $C$. albicans in some hospitals, especially in those of the southern regions [3-5].

The aim of this study was to describe the genetic pattern, and population structure, of different pathogenic Candida species recovered from blood samples, central venous catheters (CVCs), hands of healthcare workers (HCWs) and hospital environments (air, medical equipments and various surfaces) in one of the most important hospitals of Southern Italy in the treatment of patients with severe acquired brain injuries.

Materials and Methods

This study was conducted in the IRCCS Centro Neurolesi "Bonino-Pulejo" Table 1. C. albicans isolates and

hospital, Messina, Italy. All Candida isolates were initially identified using Isolates aAT1a ACC

phenotypic methods and subsequently, subjected to molecular identification IRCCS12

using species-specific PCRs and/or ITS-sequencing.

Multilocus sequence typing (MLST) method was used for $C$. albicans, $C$

glabrata and C. tropicalis genotyping (https://pubmlst.org) (Table 1-3).

our C. parapsilosis isolates. Four microsatellites markers (CP1, CP4, CP6, B1SUAP20

B5) were amplified and analyzed as described by Sabino et al., 2010 [6].

Table 2. C. glabrata isolates and multilocus sequence typing profiles generated

using seven loci of the MLST scheme.

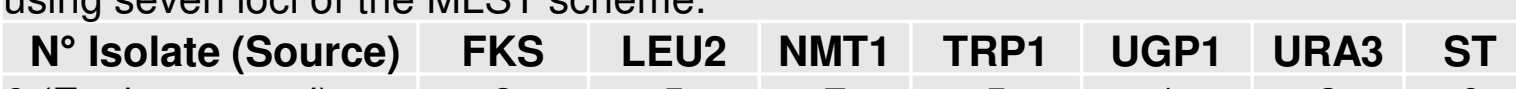

6 (Environmental)

4 (Clinical)

3 (Environmental)

\begin{tabular}{|l|l|}
\hline 2 & 5 \\
\hline 2 & 5 \\
\hline 8 & 4 \\
\hline 5 & 7 \\
\hline
\end{tabular}

\begin{tabular}{|c|c|}
\hline 7 & 5 \\
\hline 7 & 5 \\
\hline 3 & 5 \\
\hline 8 & 7 \\
\hline
\end{tabular}

\begin{tabular}{|c|c|c|}
\hline UGP1 & URA3 & ST \\
\hline 1 & 2 & 6 \\
\hline 1 & 2 & 6 \\
\hline 1 & 2 & 10 \\
\hline 3 & 6 & 3 \\
\hline
\end{tabular}

Results

BINF1V

STPA7 17

ASUAPLET17

B1S26; B4SD204

B3SD104VE

B3SD204VE

B4SD207

BINF1VE

* New DST

\begin{tabular}{|c|c|}
\hline AAT1a & ACC1 \\
\hline 62 & 12 \\
\hline 2 & 7 \\
\hline 70 & 14 \\
\hline 2 & 2 \\
\hline 2 & 3 \\
\hline 2 & 5 \\
\hline 3 & 3 \\
\hline 70 & 14 \\
\hline 60 & 13 \\
\hline 2 & 5 \\
\hline 70 & 14 \\
\hline 33 & 22 \\
\hline 62 & 13 \\
\hline 3 & 14 \\
\hline 3 & 4 \\
\hline 3 & 14 \\
\hline 96 & 7 \\
\hline 3 & 5 \\
\hline
\end{tabular}

\begin{tabular}{|c|c|}
\hline ADP1 & MPI \\
\hline 21 & \\
\hline 8 & \\
\hline 8 & \\
\hline 5 & \\
\hline 5 & \\
\hline 5 & \\
\hline 21 & \\
\hline 21 & \\
\hline 21 & \\
\hline 5 & \\
\hline 8 & \\
\hline 6 & \\
\hline 21 & \\
\hline 8 & \\
\hline 8 & \\
\hline 8 & \\
\hline 32 & \\
\hline 5 & \\
\hline
\end{tabular}

\begin{tabular}{|c|c|}
\hline MPIb & SY \\
\hline 1 & 4 \\
\hline 4 & 7 \\
\hline 4 & 7 \\
\hline 2 \\
\hline 2 \\
\hline 9 \\
\hline 1 \\
\hline 4 \\
\hline 1 \\
\hline 2 \\
\hline 4 \\
\hline 18 \\
\hline 1 \\
\hline 4 \\
\hline 26 \\
\hline 4 \\
\hline 26 \\
\hline 2
\end{tabular}

\begin{tabular}{|c|c|}
\hline SYA1 & V \\
\hline 42 & $\mid 94$ \\
\hline 7 & 10 \\
\hline 7 & \\
\hline 2 & 6 \\
\hline 2 & 6 \\
\hline 2 & \\
\hline 34 & 24 \\
\hline 7 & 30 \\
\hline 7 & 11 \\
\hline 2 & \\
\hline 42 & 6 \\
\hline 64 & 5 \\
\hline 7 & 11 \\
\hline 7 & \\
\hline 7 & 3 \\
\hline 7 & 4 \\
\hline 2 & \\
\hline 2 & 61 \\
\hline
\end{tabular}

\begin{tabular}{|c|c|}
\hline VPS13 & Z \\
\hline 194 \\
10 \\
10 \\
6 \\
6 \\
24 \\
30 \\
10 \\
11 \\
6 \\
3 \\
\hline 53 \\
11 \\
\hline 3 \\
\hline 4 \\
\hline 10 \\
61 \\
6
\end{tabular}

\begin{tabular}{|c|c|}
\hline ZWF1b & DST \\
\hline 4 & $3376^{\star}$ \\
\hline 8 & $3377^{\star}$ \\
\hline 8 & 656 \\
\hline 5 & 277 \\
\hline 20 & $3378^{\star}$ \\
\hline 5 & 185 \\
\hline 4 & $3379^{\star}$ \\
\hline 8 & $3380^{\star}$ \\
\hline 12 & $3381^{*}$ \\
\hline 5 & 69 \\
\hline 22 & $3382^{\star}$ \\
\hline 15 & $3383^{\star}$ \\
\hline 12 & $3384^{\star}$ \\
\hline 8 & $3385^{\star}$ \\
\hline 8 & $3386^{\star}$ \\
\hline 8 & $3387^{\star}$ \\
\hline 48 & $3388^{\star}$ \\
\hline 5 & 184 \\
\hline
\end{tabular}

Twenty-six blood and 28 CVCs Candida isolates were examined in this study. Two hundred-eight additional isolates (161 collected from ward environments and 47 from HCWs), were also included. C. parapsilosis (199/262; 76\%) was the most frequently isolated species followed by $C$. tropicalis (28/262; 11\%), C. albicans (21/262; 8\%) and C. glabrata (14/262; 5\%). We found a total of 28 microsatellites genotypes among 66 random selected (genotyped) C. parapsilosis isolates but only 1 genotype was shared by clinical and HCWs/environmental isolates (data not showed). MLST analysis of $C$. albicans, showed 18 different genotypes of which $13(\sim 72 \%)$ were completely new to the central MLST database (Table 1). Most of these new DSTs were found to colonize mainly hospital environments.

Genotyping of $C$. glabrata isolates showed the presence of only 3 diverse genotypes (Table 2). The genotype ST6 was the most frequent and was found in both clinical and environmental isolates. For C. tropicalis, a total of 8 different DSTs were recovered (Table 3). Interestingly, 6 (DSTs: $747-51$ and 759$)$ of these 8 genotypes (75\%) were novel to the $C$. tropicalis MLST database and one genotype (DST747) was the most frequently observed (12/28 isolates; $42.9 \%)$ followed by the genotype DST333 (6/28 isolates; $21.4 \%)$. The goeBURST analysis showed that 4 clonal clusters (CC2, CC10, CC11 and CC33) contained all the DSTs found in this study. Four genotypes (DSTs: 747, 748, 749, 759) composed a single well-defined Italian clonal cluster (CC33) with the most commonly recovered type, the DST747, as putative founding genotype (Fig. 1).

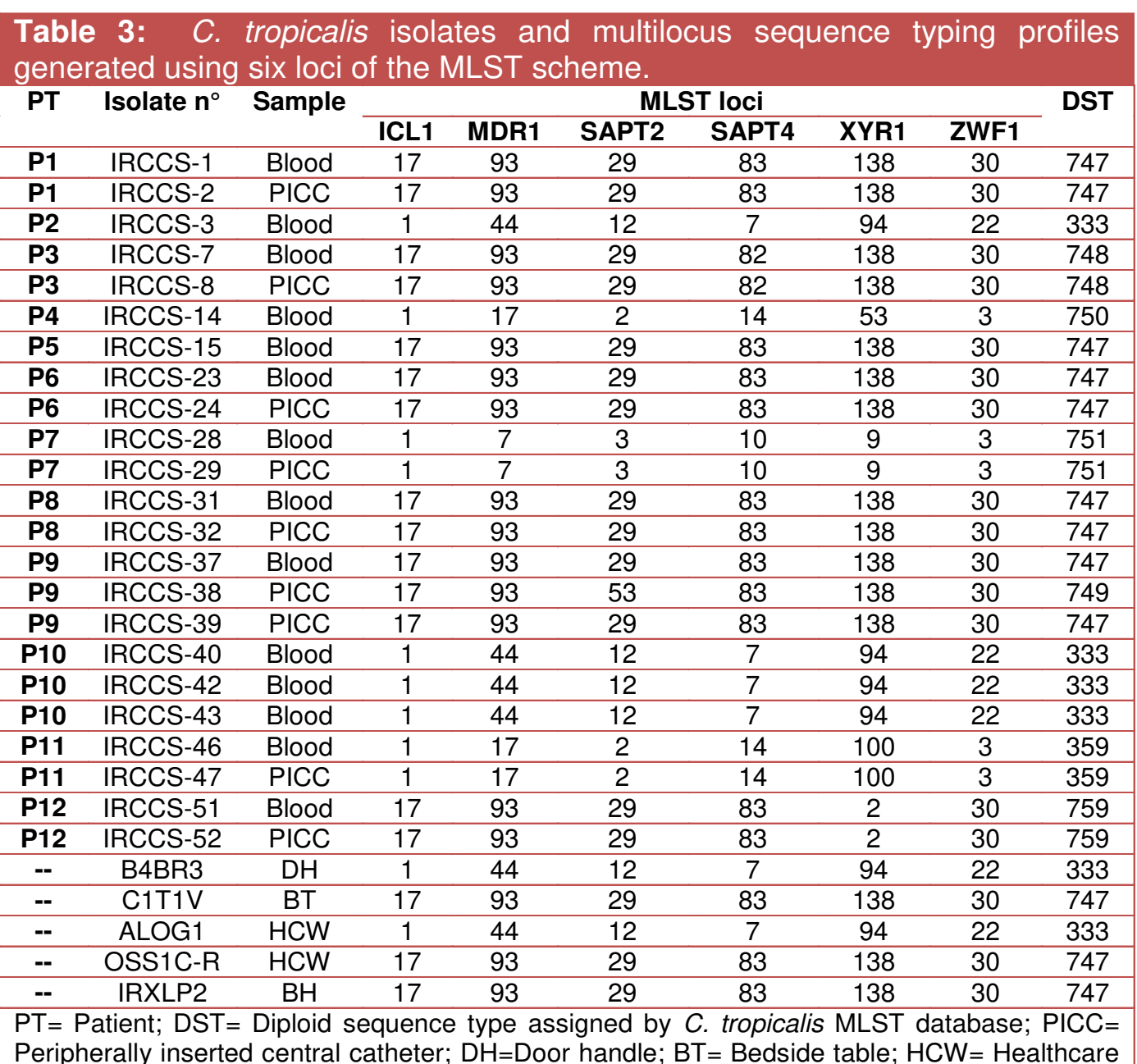

Peripherally inserted central catheter; $\mathrm{DH}=$ Door handle; $\mathrm{BT}=$ Bedside table; $\mathrm{HCW}=$ Healthcare Worker; $\mathrm{BH}=$ Bed handles.

\section{References}

1. Brown et al. doi: $10.1126 /$ scitranslmed. 3004404

2. Scordino et al. doi: $10.3389 / \mathrm{fmicb} .2018 .00679$

3. Montagna et al. doi: 10.1007/s15010-013-0432-0

4. Delfino et al. doi: $10.1111 / 1469-0691.12685$

5. Caggiano et al doi: $10.1155 / 2015 / 256580$

6. Sabino et al doi: $10.1128 / J C M .021$

7. Bassetti M, et al. doi: 10.1016/j.jinf.2017.07.008

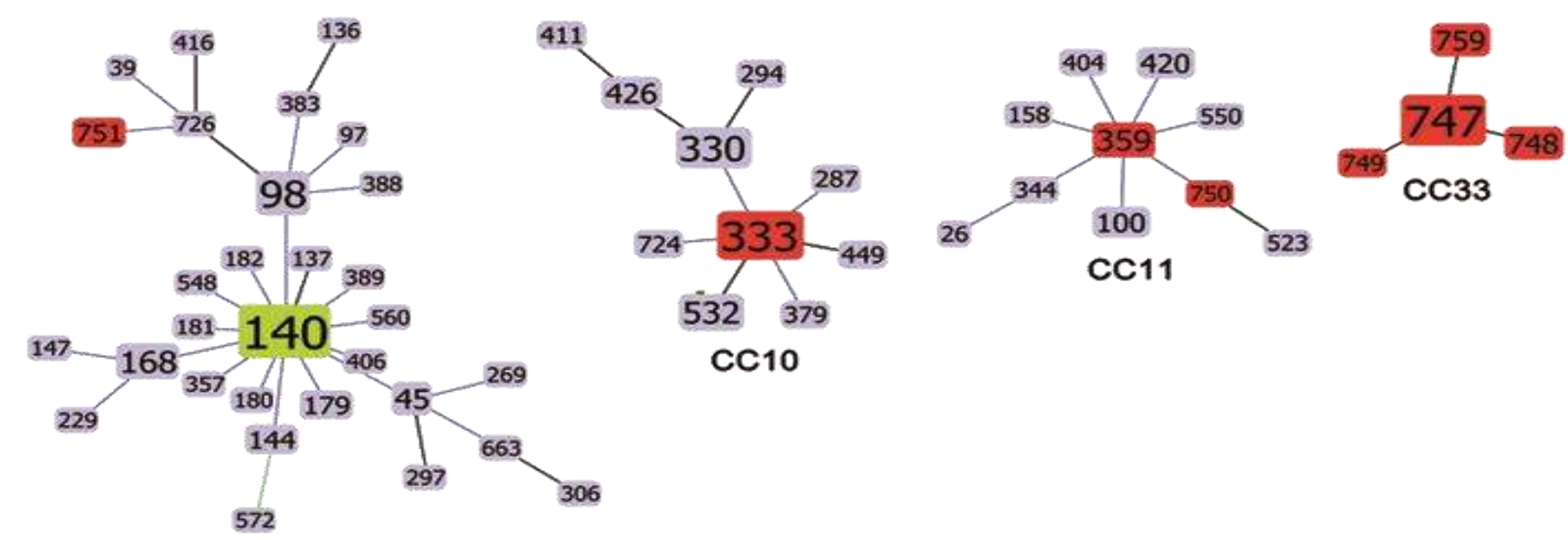

CC2 Fig. 1: goeBURST clonal clusters CC2, CC10, CC11 and CC33 containing all the DSTs (in red) found in this study. For the CC2 the putative founding genotype is indicated in green. The size of each DST reflects the number of isolates with that DST in the input data set used for the analysis.

\section{Conclusions}

Recent epidemiological data showed that in Italy candidemia is more common than in other European countries [7]. In the southern regions, a dramatic increase of BSI caused by NAC species has also been recently observed [3-5] and data from this study confirmed this trend. C. parapsilosis and C. tropicalis, appear to be the most common NAC species and outrank $C$. albicans in our and other nearby Sicilian hospitals [4].

In conclusion, this study also shows that the population structure of pathogenic Candida species is far from being fully elucidated as its complexity increases as different categories of patients and geographic areas are examined.

This work was funded by the Italian Ministry of Health (project code: GR-2011-02347606). 